\title{
Potential cancer risk with omalizumab? A disproportionality analysis of the WHO's VigiBase pharmacovigilance database
}

\author{
Diogo Mota $^{1}$, Tiago Rama ${ }^{1}$, Milton Severo ${ }^{2}$, and André Moreira ${ }^{1}$ \\ ${ }^{1}$ Centro Hospitalar Universitario de Sao Joao \\ ${ }^{2}$ Universidade do Porto Instituto de Saude Publica
}

June 29, 2021

\begin{abstract}
Potential cancer risk with omalizumab? A disproportionality analysis of the WHO's VigiBase pharmacovigilance database
\end{abstract}

\section{To the editor,}

An increasing body of evidence strongly suggests that immunoglobulin E (IgE) plays a key role in cancer immune surveillance. Moreover, a possible link between absent or very low serum IgE levels and malignancy risk has also been suggested ${ }^{1}$. Still, two studies on omalizumab, a widely used anti-IgE drug, failed to suggest any influence on the development or progression of malignancy ${ }^{2,3}$. However, one of them was based on the analysis of data from efficacy clinical trials, including carefully selected participants, enrolled for a limited period, and neither powered nor designed to assess long-term complications such as incident cancer while the other one was performed for a shorter period when compared to the latency period some solid tumors need to develop. Therefore, we aimed to examine the incidence of malignancy using comprehensive data from no selected omalizumab-treated patients recorded in a pharmacovigilance database, through assessment of a signal of cancer ${ }^{4}$.

We performed a disproportionality analysis (case/non-case study) ${ }^{4}$ within VigiBase, the World Health Organization's global database of individual case safety reports, to identify a signal of cancer, expressed as the reporting odds-ratio [ROR] and its $95 \%$ confidence interval [CI] for omalizumab. ${ }^{5}$ All Adverse Drug Reaction (ADR) reports related to omalizumab and the total number of neoplasms recorded in VigiBase were provided by the Uppsala Monitoring Centre (UMC). Cases were defined as Adverse Drug Reactions (ADR) coded as Neoplasms according to the Medical Dictionary for Regulatory Activities (MedDRA) terminology reported between 2000 and 2020. Non-cases were defined as all other ADRs during the same period. The main analysis estimated the risk of reporting "Neoplasms" associated with Omalizumab compared with all other ADRs for Omalizumab to the remaining reported drugs. Analysis for other selected cancers according to MedDRA terminology was also performed.

A total of 1380 reports mentioned neoplasms associated with omalizumab. The disproportionality signal was significant and positive: ROR $[95 \% \mathrm{CI}]=1.65$ [1.56 to 1.74]. (Table 1). Some of the most reported and with more statistical significance malignancies are presented in table 1 . The association was particularly strong in breast and lung cancer, with 232 cases and ROR [95\% CI $]=4.12$ [3.61-4.69] and, 85 cases and ROR [95\%CI] $=3.04[2.45-3.76]$ respectively (Table 1 ). Other neoplasms with strong association included prostate cancer, colon cancer, malignant melanoma, thyroid cancer and leukemia (Table 1). At the time data from VigiBase were provided, a total of more than 21 million reports were recorded in VigiBase (Figure 1).

The finding that omalizumab increases by two-thirds the risk of incident cancer is of major relevance but should be interpreted with caution. Disproportionality analysis is exploratory in the context of signal detection, not allowing quantification of the true risk. These studies have been shown to detect early signals that 
later are confirmed by controlled studies. This method has been previously used with several drugs, namely, pioglitazone, which has shown the ability to detect an early signal for cancer, later confirmed by retrospective and prospective cohorts. ${ }^{6}$. As such, a confirmatory study is needed to ascertain our findings. Information present in VigiBase comes from a variety of sources and the probability that the suspected adverse effect is drug-related is not the same in all cases. Also, VigiBase does not provide demographic data, other than gender on only some of the cases, which would allow a more accurate analysis of the risk. Duration of therapy with Omalizumab before the report of cancer is not recorded in the database. Additionally, under-reporting may have occurred. However, when referring to serious events, like cancer, this is less prone to happen and, if it did, would increase the strength of the signal. Similarly, the notoriety effect cannot apply as former studies failed to show any association between omalizumab and cancer, and it could lead to an overestimation of ROR.

Our study has important strengths. Our analysis was based on the most global and comprehensive pharmacovigilance database. Moreover, the safety of omalizumab was evaluated using post-marketing surveillance data overcoming the limitations of clinical trials inclusion criteria and limited follow-up. In conclusion, using real-world data mining, we reported a signal that omalizumab may be associated with a significantly higher risk of malignancies. Until further studies confirm the long-term safety of omalizumab, this observation should be reflected on the benefit-risk assessment when considering drugs targeting $\operatorname{IgE}$ for the treatment of allergic conditions.

\section{References}

1. Ferastraoaru D, Bax HJ, Bergmann C, et al. AllergoOncology: Ultra-low IgE, a potential novel biomarker in cancer - A Position Paper of the European Academy of Allergy and Clinical Immunology (EAACI). Clin Transl Allergy . 2020;10(1):1-16. doi:10.1186/s13601-020-00335-w

2. Busse W, Buhl R, Fernandez Vidaurre C, et al. Omalizumab and the risk of malignancy: Results from a pooled analysis. J Allergy Clin Immunol . 2012;129(4):983-989.e6. doi:10.1016/j.jaci.2012.01.033

3. Long A, Rahmaoui A, Rothman KJ, et al. Incidence of malignancy in patients with moderateto-severe asthma treated with or without omalizumab. J Allergy Clin Immunol . 2014;134(3). doi:10.1016/j.jaci.2014.02.007

4. Faillie JL. Case-non-case studies: Principle, methods, bias and interpretation. Therapie . 2019;74(2):225232. doi:10.1016/j.therap.2019.01.006

5. Montastruc JL, Sommet A, Bagheri H, Lapeyre-Mestre M. Benefits and strengths of the disproportionality analysis for identification of adverse drug reactions in a pharmacovigilance database. Br J Clin Pharmacol . 2011;72(6):905-908. doi:10.1111/j.1365-2125.2011.04037.x

6. Faillie JL, Petit P, Montastruc JL, Hillaire-Buys D. Scientific evidence and controversies about pioglitazone and bladder cancer: Which lessons can be drawn? Drug Saf . 2013;36(9):693-707. doi:10.1007/s40264-0130086-y

\section{Authors:}

Diogo Mota ${ }^{1}$

Tiago Azenha Rama ${ }^{1,2}$

Milton Severo ${ }^{3}$

André Moreira ${ }^{1,2,3}$

1 Serviço de Imunoalergologia, Centro Hospitalar Universitário de São João, Porto, Portugal;

2 Serviço de Imunologia Básica e Clínica, Faculdade de Medicina da Universidade do Porto, Portugal; 
3 EPIUnit, Instituto de Saúde Pública, Universidade do Porto, e Laboratório para a Investigação Integrativa e Translacional em Saúde Populacional (ITR), Porto, Portugal

Corresponding author:

André Moreira

andremoreira@med.up.pt

EPIUnit, Instituto de Saúde Pública, Universidade do Porto, e Laboratório para a Investigação Integrativa e Translacional em Saúde Populacional (ITR), Porto, Portugal

\section{Acknowledgements}

The information does not represent the opinion of the Uppsala Monitoring Centre (UMC) or the World Health Organization.

\section{Declaration of Competing Interest}

All authors disclose no financial and personal relationships with other people or organizations that could inappropriately influence their work.

\section{Contributors}

DM drafted the manuscript. AM conceived, and coordinated subsequent edits and revisions. MS and TR participated in drafting the manuscript and its finalization. All authors have read and approved the final manuscript.

Table 1 Disproportionality analysis (reporting odds ratio [ROR] and its $95 \%$ confidence interval $[\mathrm{CI}]$ ) of selected cancers and total neoplasms for omalizumab in VigiBase for the period between 2000 and 2020 according to gender.

\begin{tabular}{lllll}
\hline Neoplasm & Cases associated with Omalizumab, $\mathrm{n}$ & Total cases, $\mathrm{n}$ & $\mathrm{ROR}$ & $95 \%$ CI \\
\hline Breast cancer & 232 & 34449 & 4.12 & $3.61-4.69$ \\
Male & 2 & 285 & 5.94 & $1.48-23.9$ \\
Female & 204 & 31917 & 3.41 & $2.97-3.92$ \\
Lung cancer & 85 & 17012 & 3.04 & $2.45-3.76$ \\
Male & 28 & 6858 & 3.46 & $2.38-5.01$ \\
Female & 52 & 7568 & 3.66 & $2.78-4.80$ \\
Prostate cancer & 57 & 13625 & 2.54 & $1.96-3.30$ \\
Colon cancer & 46 & 7595 & 3.68 & $2.76-4.92$ \\
Male & 18 & 3222 & 4.74 & $2.98-7.53$ \\
Female & 25 & 3614 & 3.68 & $2.48-5.45$ \\
Malignant melanomal & 45 & 8258 & 3.31 & $2.47-4.44$ \\
Male & 14 & 3472 & 3.41 & $2.02-5.77$ \\
Female & 26 & 3596 & 3.85 & $2.61-5.66$ \\
Thyroid cancer & 22 & 3841 & 3.48 & $2.29-5.30$ \\
Male & 2 & 771 & 2.19 & $0.55-8.77$ \\
Female & 19 & 2364 & 4.28 & $2.72-6.72$ \\
Leukemia & 21 & 4604 & 2.77 & $1.80-4.25$ \\
Male & 9 & 1928 & 3.95 & $2.05-7.61$ \\
Female & 9 & 2060 & 2.32 & $1.20-4.46$ \\
Neoplasms (total) & 1380 & 515120 & 1.65 & $1.56-1.74$ \\
Male & 415 & 186876 & 1.91 & $1.73-2.11$ \\
Female & 868 & 253586 & 1.84 & $1.72-1.97$ \\
\hline
\end{tabular}


ROR: Reporting odds ratio; CI: confidence interval; total cases include male, female and non-specified gender. Figure 1 Flow chart of the study. ADR, adverse drug reaction; MedDRA - Medical Dictionary for Regulatory Activities 